\title{
Sistemas de Informação versus usuários
}

\author{
Celso Luiz de Souza ${ }^{1}$, Cayley Guimarães ${ }^{2}$ - orientador \\ ${ }^{1}$ celsols@yahoo.com.br, ${ }^{2}$ profcayley@yahoo.com.br \\ Curso de ciência da computação, Uni-BH - Centro Universitário de Belo Horizonte
}

\begin{abstract}
Resumo - A sociedade do conhecimento alterou as atividades humanas, aumentando os momentos em que a interação com os sistemas se tornou imprescindivel. Desenvolvedores e usuários que aprendem as novas tecnologias para realizar tarefas elou melhorar a qualidade de vida não incorporaram atitudes permissivas ao processo de usabilidade. Aos usuários são imputados os problemas dos sistemas. Os usuários, por sua vez, aceitam esta atitude e não reportam problemas de usabilidade. Este estudo baseou-se no UTAUT para testar duas hipóteses sobre essa atitude: com os usuários e com os desenvolvedores de sistema. Resultados exploratórios iniciais mostram que a usabilidade não é implementada por falta de feedback usuário/desenvolvedor..

palavras-chave - usabilidade, desenvolvimento, feedback
\end{abstract}

Abstract - The information and Knowledge society has altered human activities, increasing the arena where human-system interactions are mandatory. Designers and users that had to learn new technologies to perform some task, and/or improve their quality of life not incorporated correct attitudes towards the usability process. The users are deemed as responsible for the system's faults. And the users accept such imposition, and do not report usability problems they experience. This reseach uses the Unified Theory of Acceptance and Use of Technology (UTAUT) to test two hypothesis about this undesired attitude with the users and the designers of the system. It was found that usability is not implemented because of lack of feedback between user and designer.

keywords - usability, develop, feedback

\section{INTRODUÇÃ̃o}

A Sociedade atual, dita da Informação e do Conhecimento, altamente dependente das Tecnologias da Informação e Comunicação (TIC) vem alterando todas as atividades humanas. Os sistemas de informação são vetores de difusão da informação e inclusão, úteis em diferentes aspectos. Os usuários se deparam cada dia mais com situações de interação com sistemas seja para realizar uma tarefa, obter uma informação, enfim, tomar parte nesta sociedade da informação. Este aumento de encontro usuário-sistema propiciou um aumento da importância da usabilidade dos sistemas para tornar a experiência do usuário mais eficiente e satisfatória (Carroll 1994; Carroll, 2003). Normalmente, os usuários se deparam com situações de usar novas tecnologias e torna-se necessário aprende-las.

$\mathrm{Na}$ maioria das vezes, os desenvolvedores destes sistemas são conhecedores de tecnologias anteriores, e também têm que aprender e usar novas tecnologias no desenvolvimento.

O que se tem observado é um encontro entre estes dois grupos, materializado na interação: o desenvolvedor, detentor da técnica, pouco ou quase nunca se preocupa com a usabilidade; e, ao usuário é imposto de que o sistema é correto, e que ele (o usuário) é que está errado (Guimarães, 2008b).

Esta pesquisa procura compreender os motivos deste desencontro no processo de desenvolvimento de usabilidade. Baseado no UTAUT (Venkatesh, Morris et al., 2003) pesquisamos os desenvolvedores de sistemas de mainframe para averiguar a hipótese de que a usabilidade não é implementada porque o desenvolvedor não se preocupa com a mesma. 
Posteriormente, pesquisamos usuários para averiguar a hipótese de que a usabilidade não é implementada porque o usuário não reporta "feedbacks", ou seja, os erros de usabilidade encontrados nos sistemas não são reportados aos desenvolvedores.

\section{SOCIEDADE DA INFORMAÇÃO}

As novas tecnologias estão mudando a forma como os usuários se comunicam, interagem e obtém informações.

Neste aspecto, a usabilidade destas tecnologias desempenha um grande papel, facilitando as realizações de tarefas indispensáveis, e, conseqüentemente, o melhor exercício da cidadania(Santana e Guimarães, 2006).

De forma geral, um Sistema de Informação (SI) pode ser definido como um sistema automatizado capaz de coletar, recuperar, processar, transmitir, armazenar e disseminar dados e informações. Os SI apresentam funções técnicas ou administrativas que fazem parte do dia-a-dia das pessoas, e, portanto, o sucesso de um SI está diretamente ligado à sua integração com o ambiente social em que ele se insere (Laudon e Laudon, 2002).

Segundo Sorj (2003) as novas tecnologias devem ser úteis e apresentar uma boa interatividade com os seres humanos, permitindo uma integração social e potencializando a qualidade de vida. Neste aspecto, o autor destaca, dentre os SI, a Internet, como sendo uma fonte eficaz de conhecimento para as pessoas, capaz de suprir falhas e complementar os meios de comunicação tradicionais. Para (Sorj, 2003), a Internet promove a cidadania: aumentando a qualidade, transparência e fiscalização, sobretudo das ações e serviços do governo e das instituições públicas; incrementando novas formas de participação e influência nas gestões; e modificando a própria estrutura das organizações políticas da sociedade. Este conceito de integração homemmáquina se mistura à idéia de usabilidade (Nielsen, Bush et al., 1992; John, 1994; Carroll, 2003).

\section{SOCIEDADE DA INFORMAÇÃO E USABILIDADE}

As Tecnologias da Informação e Comunicação alteraram vários aspectos das atividades humanas, entre eles o trabalho, o aprendizado e o uso de tecnologia em geral. Estas mudanças são amplas e profundas a ponto de serem entendidas como um novo paradigma técnico-econômico: os usuários utilizam cada vez mais tecnologia no dia-a-dia, para um gama de tarefas.

Os sistemas demandados pelos usuários desta nova sociedade devem atender às características de Interface Homem Máquina (IHC), Fatores Humanos e Usabilidade. A falta de usabilidade pode coibir o uso adequado da ferramenta para o 
objetivo desejado, segundo Santana e Guimarães (2006), com prejuízos à sociedade.

Comumente a IHC é ensinada com teorias e regras. Mas esta abordagem tradicional não apresenta os resultados esperados. Nielsen (Nielsen, Bush et al., 1992; 1999) associa IHC a cinco atributos: Facilidade de Aprendizado, Eficiência, Facilidade de Relembrar, Prevenção de erros e Satisfação subjetiva. Embora estes atributos pareçam simples, na prática os desenvolvedores e os sistemas não os seguem, causando todos os tipos de problemas ao usuário. A necessidade de se estudar Fatores Humanos e Usabilidade surgiu da observação do embate diário dos usuários nas suas interações e problemas com sistemas para realizar alguma tarefa (Tonini, 2007; Guimarães, 2008b; a).

Esses problemas são decorrentes de desenvolvedores altamente capacitados tecnicamente, mas pouco informados sobre as necessidades do usuário. Desenvolvedores que, ao se concentrarem nos aspectos técnicos, deixam de lado os aspectos da interação do sistema com o usuário, a ponto de culpar exclusivamente o usuário pelas causas dos problemas. Uma mudança de atitude é um bom ponto de partida. Atitude do usuário, em não aceitar sistemas inadequados. Atitude dos desenvolvedores de acharem que a tecnologia, por si, basta. E esta mudança só será possível sensibilizando os desenvolvedores para além da técnica e voltando-os para o usuário.

O profissional tem que ter sempre em mente que na comparação de custos de hardware \& software vs. Usuários, definitivamente os componentes de hardware e software são os mais baratos, os dispensáveis. O usuário é o mais importante. Temos observado muitos sistemas bons tecnicamente, mas ruins na interação, ou seja, eles podem até apresentar uma correção técnica do ponto de vista de funcionalidade, mas deixam muito a desejar quanto ao uso pelo usuário.

O grande problema com que nos deparamos é o de garantir, no design de novos sistemas, usabilidade (Nielsen, 1999). Ao analisarmos a IHC, percebemos que a maioria dos sistemas não foram projetados pensando no usuário usando o sistema para realizar uma tarefa, mas projetados com várias funcionalidades que viriam a servir o usuário.

No entanto, quando os desenvolvedores acertam, criando sistemas que enriquecem a nossa experiência, nos auxiliam em nossas tarefas, são simples de usar, alçamos vôos em suas visões. Mas quando erram, restam-nos os pesadelos. IHC é determinável, quantificável. Portanto, pode ser deliberadamente construída e testada.

Estes sistemas se comportam mal porque são mal projetados. O que não significa que não 
existam boas idéias nem habilidades técnicas que foram utilizadas em seu design, mas sim dizer que as boas idéias talvez estejam soterradas por idéias ruins no meio do processo confuso de design desestruturado. Existem excelentes idéias escondidas no design da maioria dos sistemas, mas o conjunto acaba saindo bem menor do que a soma das partes. (Carroll 1994; Carroll, 2003; Guimarães, 2008a).

Contudo, apesar da disseminação destes sistemas tecnológicos complexos vir aumentando nas últimas décadas e de termos diversos trabalhos pioneiros na área (Reitman, 1965; Schön, 1967; Scriven, 1967; Carroll, 2003), apenas recentemente os desenvolvedores de sistemas começaram a retomar a preocupação com os aspectos da interação destes sistemas com o usuário.

Todo e qualquer sistema foi feito para servir ao usuário, parte mais importante do processo. $\mathrm{E}$ é esta tecnologia que deve se adequar ao usuário, e não o contrário. $\mathrm{O}$ erro encontra-se justamente na inadequação que ocorre entre o design, o sistema e o usuário. Surge a necessidade de mostrar, tanto para o usuário quanto para o desenvolvedor, que, se um sistema não está atingindo o seu objetivo, uma análise adequada e isenta deve ser feita. Na maioria das vezes, esta análise mostra que o problema encontra-se no projeto e na ferramenta, e não no usuário. Infelizmente é muito comum ouvir, até mesmo dos próprios usuários, que eles é que são os culpados, os incompetentes. Esta é a mudança de atitude que se deve enfatizar.

\section{Modelos de ACEITAÇão de TeCnOlogia}

Apesar desta importância crescente, para que a tecnologia realmente cause impacto, ela tem que ser aceita e usada pelas pessoas. As pesquisas nesta área geraram diversos modelos que explicam a intenção de uso de tecnologia (Fred, Richard et al., 1989; Venkatesh e Davis, 2000). Veja a seguir alguns exemplos destes modelos

- A Teoria do Comportamento Racional (TRA) é uma delas, usada para explicar diversos comportamentos; considerando a atitude em relação ao comportamento e normas subjetivas (Fred, Richard et al., 1989).

- Venkatesh e Davis (2000) incorporando percepção de utilidade e facilidade de uso em relação à TRA, o Modelo de Aceitação de Tecnologia (MAT) é mais adaptado a sistemas de informação.

- O Modelo Motivacional (MM), adaptado para o domínio em estudo, tem sido bastante usado (Vallerand, 1997).

- A teoria de comportamento planejado é uma extensão do TRA, ao adicionar a variável de 
percepção de controle de comportamento (Ajzen, 1991).

Neste estudo iremos nos valer da teoria unificada de aceitação e uso de tecnologia (UTAUT), proposta por Venkatesh (2003). Mais especificamente, usaremos como pano de fundo quatro das variáveis do modelo (UTAUT) (Venkatesh, Morris et al., 2003):

- Expectativa de Performance: definida como sendo o grau com que um indivíduo acredita que o uso do sistema irá ajudar a atingir ganhos de performance no trabalho.

- Expectativa de Esforço: definida como sendo o grau de facilidade associado com o uso do sistema.

- Influência social: definida como sendo o grau de percepção de um indivíduo de que outras pessoas importantes acreditam que ele deva usar o novo sistema.

- Condições Facilitadoras: definida como sendo o grau de percepção do indivíduo de que existam estruturas técnicas e organizacionais que suportam o uso do sistema.

Estas variáveis, combinadas com a nossa discussão sobre a atitude dos desenvolvedores de imputarem ao usuário a culpa pela falta de usabilidade, e a atitude dos usuários em aceitar esta imposição.
Este estudo apresenta uma pesquisa exploratória desenvolvida com o objetivo de proporcionar visão geral acerca de que após constatarmos os problemas de usabilidades através de uma Avaliação Heurísticas, procuramos averiguar o motivo pelo qual os desenvolvedores não implementaram a usabilidade. Este tipo de pesquisa é realizado especialmente quando o tema escolhido é pouco explorado e torna-se difícil sobre ele formular hipóteses precisas e operacionalizáveis. Segundo Ariboni \& Perito (2004) a pesquisa exploratória serve ao propósito de desenvolver, esclarecer e modificar conceitos e idéias, com vistas à formulação de problemas de estudo mais precisos ou hipóteses pesquisáveis para estudos posteriores. A seguinte presunção foi elaborada: Presunção 1: A usabilidade não é implementada porque os desenvolvedores não levam em conta a verificação, junto ao usuário, de problemas de usabilidade.

Presunção 2: A usabilidade não é implementada porque o usuário não reporta (feedback), os erros de usabilidade encontrados no sistema.

\section{Pesquisa}

Trata-se de um estudo de caso exploratório, desenvolvido com o objetivo de avaliar o motivo pelo qual os desenvolvedores não implementam a usabilidade. Este tipo de pesquisa é indicado quando o tema escolhido é pouco explorado e 
torna-se difícil sobre ele formular hipóteses precisas e operacionalizáveis.

Segundo Ariboni \& Perito (2004), a pesquisa exploratória serve ao propósito de desenvolver, esclarecer e modificar conceitos e idéias, com vistas na formulação de problemas de estudo mais precisos ou hipóteses pesquisáveis para estudos posteriores.

\section{Parte I}

Esta pesquisa foi realizada em duas partes. Primeiro os autores realizaram uma Avaliação Heurística do sistema acadêmico. A avaliação seguiu os procedimentos e heurísticas propostos por Nielsen(1999):

H1. Visibilidade do estado do sistema;

H2. Correspondência entre o sistema e o mundo real;

H3. Controle do usuário e liberdade;

H4. Consistência e padrões;

H5. Prevenção de erros;

H6. Ajuda ao usuário a reconhecer, diagnosticar e se recuperar de erros;

H7. Reconhecimento ao invés de lembrar;

H8. Flexibilidade e eficiência de uso;

H9. Design e estética minimalista.

H10. Ajuda e documentação.

Cada problema achado foi analisado quanto aos seguintes fatores:

- Frequiência (problema comum ou raro);

- Impacto (qual a conseqüência);
- Persistência (ocorre uma vez);

- Impacto de mercado (afeta a credibilidade);

Estes fatores foram analisados e combinados em uma escala de severidade: 0 - Problema cosmético; 1 - Pequeno problema de usabilidade; deve ser resolvido com baixa prioridade; 2 - Grande problema de usabilidade; deve ser resolvido com alta prioridade; e 3 Catástrofe de usabilidade; é imperativo que seja corrigido.

A avaliação foi realizada do ponto de vista do estudante, formando do curso de Ciência da Computação da instituição, com grande experiência de uso do sistema, realizando tarefas rotineiras (e.g. verificar notas e faltas, solicitar histórico, etc.).

\section{Parte II}

Após a avaliação heurística, foram entrevistados sete analistas que participaram do processo de desenvolvimento do sistema.

Os pesquisados são homens e mulheres com idade entre 28 e 34 anos. Todos os pesquisados possuem formação superior: quatro em Ciência da Computação, dois em Administração e um em Engenharia Elétrica. $\mathrm{O}$ objetivo desta entrevista era constatar a razão pela qual o sistema apresentava os problemas de usabilidade. Mais precisamente, averiguamos a seguinte hipótese: 
Presunção $1-A$ usabilidade não é Para tanto, foram feitas as seguintes perguntas: implementada porque o desenvolvedor não se preocupa com a satisfação do usuário.

Para tanto, foram feitas as seguintes questionamentos:

1) O desenvolvimento de software não leva em consideração a verificação, junto ao usuário, dos problemas de usabilidade.

2) Não há necessidade de se verificar a satisfação por parte do usuário, pois o próprio desenvolvedor deve atentar para os problemas de usabilidade do sistema, durante o desenvolvimento do mesmo.

3) Entrevistas com o usuário são necessárias somente quando há erros de funcionalidade no sistema, que ocorrem ainda durante o desenvolvimento do mesmo.

4) Informações dadas pelo usuário não dão ao desenvolvedor subsídios adicionais para melhorar a usabilidade do sistema.

5) A usabilidade não é dependente de verificação. O desenvolvedor deve atentar para erros, mas não deve depender de respostas dos usuários para melhorar o sistema.

Presunção $2-A$ usabilidade não é implementada porque o usuário não reporta (feedback) os erros de usabilidade encontrados 1) A usabilidade não é implementada porque o usuário não reporta os erros de usabilidade encontrados no sistema.

2) Não há necessidade de feedback por parte do usuário, pois o próprio desenvolvedor deve atentar para os erros do sistema.

3) Feedbacks são necessários somente quando há erros de funcionalidade no sistema.

4) Feedbacks feitos pelo usuário não dão ao desenvolvedor mais dados para aplicar usabilidade ao sistema.

5) A usabilidade não é dependente de feedback. O usuário não precisa reportar os problemas encontrados, pois o desenvolvedor é o responsável por garantir a usabilidade do mesmo.

Cada uma das perguntas para a hipótese foi testada usando o teste $\mathrm{t}$ de uma coleta, de um lado, com a hipótese nula sendo que a média da amostra é menor ou igual a três (neutro, ponto do meio na escala tipo Likert: 1 - Discordo Fortemente; 2 - Discordo; 3 - Neutro; 4 Concordo; 5 - Concordo Fortemente). A hipótese alternativa (a um nível de significação de 0,025 para o teste t de um lado) diz que a média da amostra é maior do que três e que os respondentes concordam com a pergunta. no sistema. 


\section{RESUltados E ANALISES}

\section{Parte I}

A avaliação heurística encontrou as seguintes quantidades de problemas de heurísticas conforme a Tabela 1, e a Tabela 2 mostra o grau de severidade.

Tabela 1 - Resultado da avaliação heurística

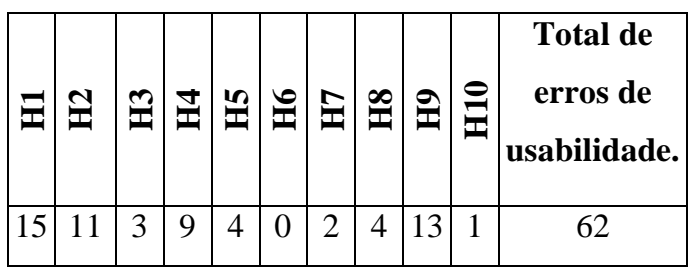

Tabela 2 - Distribuição do grau de severidade

\begin{tabular}{|l|l|l|l|l|}
\hline Grau de severidade & 0 & 1 & 2 & 3 \\
\hline Quantidade de Erros & 19 & 23 & 16 & 4 \\
\hline
\end{tabular}

\section{Parte II}

A Tabela 3 a seguir apresenta os resultados de cada pergunta da Presunção 1, levantada:

Tabela 3 - Respostas às perguntas da Presunção 1

\begin{tabular}{|c|c|c|c|c|c|}
\hline 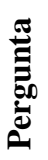 & 苞 & 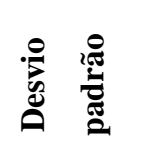 & - & 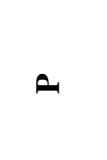 & 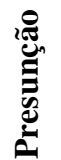 \\
\hline 1 & 2,952381 & 0,74 & 3,29 & 0,0001 & P1 \\
\hline 2 & 3,761905 & 1,135991 & 4,26 & 0,001 & P1 \\
\hline 3 & 3,619048 & 0,804748 & 3,99 & 0,0001 & P1 \\
\hline 4 & 3,047619 & 0,804748 & 3,45 & 0,0001 & P1 \\
\hline 5 & 3,047619 & 0,740013 & 3,42 & 0,0001 & P1 \\
\hline
\end{tabular}

Observa-se que na tabela 3 , pode-se concluir pela presunção 1: A usabilidade não é implementada porque o desenvolvedor não se preocupa em verificar, junto ao usuário, os problemas de usabilidade. Embora o sistema seja de uso freqüente e antigo, ainda sim foi possível detectar 62 erros, incluindo 4 erros catastróficos.

Para Presunção 2, a Tabela 4 apresenta os resultados obtidos das perguntas.

Tabela 4 - Respostas às perguntas da Presunção 2

\begin{tabular}{|c|c|c|c|c|c|}
\hline 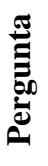 & 胥 & 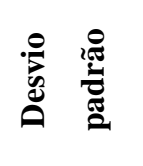 & - & a & 苞 \\
\hline 1 & 3,328461 & 0,744325 & 3,20 & 0,0001 & $\mathrm{P} 2$ \\
\hline 2 & 3,930255 & 0,935991 & 4,01 & 0,0001 & $\mathrm{P} 2$ \\
\hline 3 & 4,528413 & 0,804748 & 4,01 & 0,0001 & P2 \\
\hline 4 & 3,631492 & 0,804748 & 3,45 & 0,0001 & $\mathrm{P} 2$ \\
\hline 5 & 3,031495 & 0,741103 & 3,01 & 0,0001 & P2 \\
\hline
\end{tabular}

Como se pode notar pelos resultados apresentados na tabela 4 podemos concluir pela presunção 2, ou seja: A usabilidade não é implementada porque o usuário não reporta (feedback) os erros de usabilidade encontrados no sistema.

\section{CONCLUSÕES}

Usou-se o modelo UTAUT para verificar com os desenvolvedores de sistemas para mainframe sobre o motivo de não implementarem a usabilidade. Concluiu-se que os desenvolvedores não implementam usabilidade porque não se preocupam em verificar, junto ao usuário, os problemas de usabilidade.

Usou-se o modelo UTAUT para verificar com os usuários de sistemas sobre o motivo de 
não reportarem erros. Concluiu-se que os usuários não reportam os problemas de usabilidade com que se deparam.

Este estudo inicial mostra que, apesar dos desenvolvedores serem detentores da técnica, ainda assim eles não se sentem motivados a aplicar a usabilidade no desenvolvimento de sistemas.

Este achado indica que uma transformação nas metodologias de ensino possa ser necessária, no sentido de conscientizar o desenvolvedor e o usuário da importância crescente da usabilidade. Outros estudos, seguindo a mesma linha, devem ser realizados. Por exemplo, testar o motivo pelo qual o usuário não reporta os problemas. Uma possível pressuposição é de que ele não dispõem de um canal de comunicação para fornecer este feedback. Também, outra possível hipótese é a de que este canal de comunicação não é disponibilizado pelo desenvolvedor, pois o mesmo não dá a devida importância à usabilidade.

\section{REFERÊNCIAS}

AJZEN, I. The theory of planned behavior. Organizational Behavior and Human Decision Processes [S.I.],City, v. 50, n. 2, p. 179-211, Dez. 1991.

ARIBONI, S.; PERITO, R. Guia prático para um projeto de pesquisa exploratória, experimental, descritiva. São Paulo: Unimarco 2004.
CARROLL , J. M. Making use: a design representation. Commun. ACM [S.I.],City, v. 37, n. 12, p. 28-35, 1994.

CARROLL, J. M. HCI Models, Theories, and Frameworks: Toward a multidisciplinary science. San Francisco: Morgan Kaufmann; 1 edition (April 10, 2003), 2003. (Interactive Technologies).

FRED, D. D. et al. User acceptance of computer technology: a comparison of two theoretical models. Manage. Sci. [S.I.],City, v. 35, n. 8, p. 982-1003, 1989.

GUIMARÃES, C. Usabilidade no dia-a-dia - a interação de seres humanos com sistemas. Belo Horizonte, MG: Fundac-BH, 2008a.

Usabilidade no dia-a-dia: Novo Contexto de Ensino de IHC. In: SENEPT - I Seminário Nacional de Educação Profissional e Tecnológica, 2008b, Belo Horizonte. 2008b JOHN, M. C. Making use: a design representation. Commun. ACM [S.I.],City, v. 37, n. 12, p. 28-35, 1994.

LAUDON, J. P.; LAUDON, K. C. Sistemas de Informção Gerenciais. São Paulo, SP: Rentice Hall (Pearson), 2002.

NIELSEN, J. Design Web Usability. Indianapolis, Indiana, EUA: New Riders Publish, 1999.

NIELSEN, J. et al. Teaching experienced developers to design graphical user interfaces. In: Conference on Human Factors in Computing 
Systems Proceedings of the SIGCHI conference on Human factors in computing systems, 1992, Monterey, California. ACM, 1992. p.557-564 REITMAN, W. R. Cognition and thought: an information processing approach. New York: John Wiley \& Sons, Inc., 1965. SANTANA, M. C. L. G.; GUIMARÃES, C. Sistema de Informação e Cidadania - a falta de usabilidade pode tolher a democracia. In: 2003.

SENEPT In anais do III SBC/SBSI, 2006, Curitiba, PR. 2006

SCHÖN, D. A. Technology and change: The new Heraclitus. New York: Pergamon, 1967.

SCRIVEN, M. The methodology of evaluation. In: Perspectives of Curriculum Evaluation.TAYLOR, R. et al (Ed.). Chicago: Rand McNally, 1967. p. 39-83.

SORJ, B. Brasil@povo.com: a luta contra a desigualdade na sociedade da informação. Jorge Zahar Editor Ltda. ed. Rio de Janeiro, JR, 2003.

TONINI, A. M. Ensino de engenharia: atividades acadêmicas complementares na formação do engenheiro. 2007. dissertação, (Mestre) - Faculdade de Educação, UFMG, Belo Horizonte, MG, 2007.

VALLERAND, R. J. Toward a hierarchical model of intrinsic and extrinsic motivation. In: Advances in experimental social psychology.ZANNA, M. P. (Ed.). New York: Academic Press, 1997. Cap.271. p. 271-360.
VENKATESH, V.; DAVIS, F. D. Theoretical Extension of the Technology Acceptance Model: Four Longitudinal Field Studies. Management Science [S.I.],City, v. 46, p. 189207, Fev. 2000.

VENKATESH, V. et al. User Acceptance of Information Technology: Toward a Unified View. MIS Quarterly [S.I.],City, v. 27, n. 3, 